\title{
DE L'ENSEIGNEMENT DES EFFETS DE SENS DE CERTAINS TEMPS FRANÇAIS
}

\begin{abstract}
A bstr act. Florczak Jacek, De l'enseignement des effets de sens de certains temps français [Teaching the communicative effects of several French tenses]. Studia Romanica Posnaniensia, Adam Mickiewicz University Press, Poznań, vol. XXXI: 2004, pp. 247-257. ISBN 83-232-1353-4, ISSN 0137-2475.
\end{abstract}

The paper presents an unconventional technique to teach the communicative effects (CE) induced by the use of several French tenses in the context. This technique is constructed for Polish students whose language system does not lexicalise these $\mathrm{CE}$ like the French system. We start from the principle of the subordinated bilingualism, when the target language acquisition is performed by means of the native language and in order to "feel" the CE of the French verbal action we propose several experiences.

\section{INTRODUCTION}

A l'origine des problèmes dans l'acquisition de la compétence d'une langue étrangère se trouve le fait que l'usager d'une langue est habitué à percevoir le monde tel qu'il est conceptualisé par sa langue. Cette habitude fait non seulement qu'il lui est difficile de concevoir les concepts d'une langue étrangère, mais elle fait naître en plus le besoin de décrire le monde dans la langue étrangère tel qu'il le perçoit dans sa langue maternelle. D'une part nous conceptualisons donc les formes d'expression étrangères à la manière de notre langue, ce que prouve l'exemple désormais classique de l'assimilation de l'aspectualité slave aux valeurs des temps français qui fait que «les apprenants ont tendance à assimiler automatiquement l'imperfectif à l'imparfait et le perfectif au passé composé» (Štichauer, 2002: 272):

Nic mi nigdy nie wythmaczyt

Nic mi nigdy nie thumaczyt
Il ne m'a jamais rien expliqué

ll ne m'expliquait jamais rien

D'autre part nous cherchons à mettre en profil ce qu'exprime notre langue pour décrire ces aspects de la réalité que nous sommes habitués à considérer comme ceux qui méritent d'être exprimés, et nous avons du mal à comprendre que 
ce qu'une langue exprime comme une chose importante peut ne pas l'être pour l'autre. C'est pour cela que l'apprenant polonais cherche à exprimer en français l'opposition: naprowiat, ale nie naprawit', alors que l'apprenant français cherchera dans le polonais un moyen d'expression simple de la différence entre ce que j'ai eu vs ce que j'avais froid hier.

L'interférence des structures maternelles sur la langue cible mène finalement à affecter les expressions étrangères de valeurs qu'elles ne possèdent pas ou à enlever celles qui leur sont propres. Dans ce cas, ou bien nous interprétons mal l'effet de sens (ES) du temps employé, ou bien nous employons un temps dont l'ES est inadéquat à celui que nous voulions induire, ce qui conduit à différentes aberrations de communication, soit: à des situations où les emplois tout à fait corrects seront reçus comme surprenants, voire inacceptables:

(:) Demain (Maintenant) je suis parti

๑ J'ai perdu la montre en or depuis longtemps

*Jutro (Teraz) wyjechatem

*Zgubilem zloty zegarek od dawna

ou bien aux emplois du temps dont l'ES ne correspond pas à l'intention de l'émetteur:

( ) je n'ai pas pu jouer avec vous parce que je me suis cassé le bras - quand le bras est déjà guéri

(e ne pouvais pas jouer avec vous parce que je m'étais cassé le bras - quand le bras est encore dans le plâtre

\section{APPROCHE CONFRONTATIVE}

Les linguistes savent très bien que chaque langue conceptualise sa réalité à sa manière et qu'il n'est pas possible de projeter directement les structures conceptuelles d'une langue sur une autre. Outre les différentes conceptualisations d'un phénomène (p.ex.: padat deszcz vs il a plu), il se peut également que ce qu'une langue mette en profil, l'autre n'en rende pas compte (p.ex.: je te l'avais dit vs a nie mówiłem), et les formes d'expression conventionnalisées dans une langue peuvent être considérées comme celles qu'une autre langue n'a pas l'habitude d'exprimer (p.ex.: I'm reading vs Jestem $w$ trakcie czytania), et même si elles peuvent être considérées comme "admissibles», elles seront perçues comme non conventionnelles, voire incorrectes (p.ex.: :) J'ai oublié ma montre vs $\odot \mathrm{Mam}$ zapomniany zegarek).

Les différences relatives à l'expression de l'action verbale (AV) en polonais et en français, ou, plus largement, en slave et en roman sont bien connues. E. Coseriu constate: «En slave, on exprime l'action verbale en tant qu'envisagée avec son terme objectif ou sans son terme, tandis qu'en roman on exprime l'action avec des

'Il s'agit d'une possibilité «grammaticale», quoique même une traduction périphrastique de la phrase polonaise ci-dessus: $j$ 'ai tenté de le réparer, mais je n'y suis pas arrivé rajoute certaines significations et en omet d'autres. 
limites définies dans le temps ou sans limites, ou, pour mieux dire, l'action en dehors de son déroulement et dans son déroulement même» (d'après: Štichauer, 2002: 273).

En français, dominent donc les relations temporelles (antériorité, postériorité, optique: «maintenant» $v s$ «alors», bref, une dizaine de temps grammaticaux différents), alors que le polonais, lui, accorde beaucoup d'importance à la catégorie de l'aspect, qui, étant grammaticalisée, le fait choisir, à chaque partie du discours à caractère déverbal, entre le perfectif et l'imperfectif, et percevoir son action comme achevée ou non (sa temporalité se borne aux trois temps les plus simples).

Bref, l'AV française est conceptualisée de manière à mettre en profil la valeur temporelle, tandis que dans l'AV polonaise on met en profil la valeur aspectuelle; si donc la première n'est pas tellement importante pour l'usager de la langue polonaise, le français ne cherchera pas tellement à exprimer la seconde ${ }^{2}$.

La perception de l'AV en français et en polonais est donc bien différente, et l'apprenant polonais devrait comprendre que, par exemple, le PC français n'est pas synonyme du passé perfectif polonais, mais qu'il a ses propres valeurs. Alors une question apparaît: si leurs structures conceptuelles sont tellement différentes et que l'élève ne perçoit le monde que par le biais de la / sa langue, comment enseigner les concepts qui lui sont étrange(r)s, alors qu'il ne dispose que des concepts de sa langue maternelle? C'est pour faire sentir certaines d'entre ces valeurs de l'AV française que notre technique a été conçue.

\section{APPROCHE PRAGMATIQUE}

Toute expression du temps (T) peut être analysée dans différentes perspectives $^{3}$ et les valeurs de l'action verbale peuvent être de différentes natures. Nous focaliserons notre attention sur sa valeur pragmatique. Dans cette perspective, l'emploi du T en contexte sera perçu comme un outil de communication reliant les acteurs de l'échange langagier par ses divers effets de sens ou les effets de son

${ }^{2}$ Nous ne nous engageons pas dans la querelle entre ceux qui considèrent le français comme non aspectuel, car il n'a pas formé de morphèmes aspectuels grammaticaux à l'instar des morphèmes aspectuels slaves et ceux qui lc classifient comme langue aspectuelle, l'aspect étant considéré comme trait inhérent du sens des verbes mêmes, soit des morphèmes lexicaux (p.ex.: duratif: connaître, inchoatif: concevoir; Gross, 2002: 1991) ou bien des énoncés formés à la base de ces verbes, (p.ex.: $I l$ est mort; Štichauer, 2002: 70). Nous considérons pour des raisons de simplicité qui est indispensable pour un apprenant non initié en linguistique que les traits d'inchoativité, de durativité, etc. attribués aux verbes français ne décrivent pas l'aspect à la polonaise (d'ailleurs, l'opposition: perfectivité vs imperfectivitê n'est pas toujours comprise de la même manière dans le polonais et dans le français; $\mathrm{cf}$. p.ex. Langacker, 1991, Turewicz, 1998) mais les ordres de procès qui se trouvent aussi bien dans les verbes français que dans les verbes polonais (ex.: inchoatif: poczqć, duratif: $z n a c ́$ ).

${ }^{3}$ Au niveau syntactique seront formulées les règles qui conditionnent son emploi dans différentes constructions, comme: je te le (FS)rendrai a p rès que je l'(FA)aurai terminé; sur le plan stylistique seront expliquées les oppositions du type: nous voulions vs nous voulûmes, etc. 
emploi relativement aux inférences qu'il induit dans une situation de communication donnée. L'exemple de telles inférences peut être fourni par les phrases suivantes:

Si elle avait été riche... $\rightarrow$ (implique) |elle ne l'est pas $\mid$

Ce qu'elle était riche! $\supset$ (sous-entend) | elle ne l'est plus |

Pour comprendre la notion d'effet de sens on remarquera que le locuteur, au moment où il envisage la possibilité d'une certaine parole, se représente dans le même temps l'effet éventuel de son emploi ou les conséquences que l'auditeur en tirera et il aura tendance à choisir les paroles qui promettent de produire les effets qu'il désire. (Ducrot, 1972: 15) La situation qui aura lieu suite à l'emploi d'une expression s'appellera l'effet de son emploi (Tokarz, 1993: 253). Dans les exemples ci-dessus, les ES des temps IMP et PC s'expriment à droite de l'énoncé (y compris leur statut logique d'implication et de sous-entendu), et l'effet de leur emploi peut produire des conséquences diverses, dont l'une pourrait être, pourquoi pas, de prendre la décision de ne pas se marier avec la fille dont il est question dans les phrases ci-dessus.

Dans cette approche pragmatique, l'emploi du $\mathbf{T}$ relève d'un choix du locuteur en vue d'induire tel ou tel ES chez l'auditeur à partir des inférences établies dans un contexte, et l'analyse de son emploi ne se fait pas en termes d'emploi (in) correcte ( $p$ vs ${ }^{*} p$ ), mais en ceux d'emploi (mal)approprié (in/acceptable) ou (in)efficace, bref, en termes d'emploi réussi (:) ou pas $(*)$. Nous dirons que l'emploi du $\mathrm{T}$ est approprié (acceptable) si et seulement si les conditions inférencielles de son emploi sont remplies. Nous dirons que l'emploi du T est efficace, lorsque l'effet de son emploi correspond à l'objectif visé, i.e. lorsque l'ES qu'il induit chez l'auditeur est conforme aux intentions du locuteur. En termes plus précis: si en disant $\alpha$ nous voulions donner lieu à la situation $v$, et qu'il s'est établi la situation $\varpi$, alors notre énoncé, où nous avons employé tel ou tel temps, a été efficace si et seulement si $v=\varpi$ (cf. Tokarz, 1993: 256). En revanche, lorsque les conditions de l'emploi du $\mathrm{T}$ ne sont pas remplies et que l'intention du locuteur n'est pas atteinte, l'emploi du $\mathrm{T}$ sera considéré comme étant, respectivement, mal approprié ou inefficace, et l'énoncé mal réussi. P.ex. les phrases:

* Il m'a rendu la cassette que je lui ai empruntée; cond. inférencielles non remplies

(: Il ne m'a pas rendu la cassette que je lui avais empruntée; cond. infér. non remplies

(8) Je suis venu en retard parce que j'ai oublié ma montre - et je la regarde; $v \neq \omega$

(*) Je suis venu en retard parce que j'avais oublié ma montre - et je ne l'ai toujours pas; $v \neq \varpi$

seront considérées comme mal réussies, cat on ne peut pas avoir et ne pas avoir (emprunté ou oublié qqch) à la fois. Si donc le choix du $T$ relève du locuteur, sans savoir ce que ce $T$ veut dire il risque de dire ce qu'il ne voulait pas dire, et l'objectif de l'enseignant devrait être de trouver des techniques d'appropriation des ES des T français qui permettent à l'apprenant polonais de les employer de manière à ce que son auditeur (n')ait (pas) compris ce qu'il (n')a (pas) voulu qu'il comprenne. 


\section{APPROCHE DIDACTIQUE}

Notre méthode se compose de deux étapes réalisées parallèlement. Dans la première, nous sensibilisons les élèves aux différents moyens de conceptualisation et de mise en profil de l'action verbale dans les deux langues, en vue de les déshabituer à percevoir la réalité linguistique du français à la manière polonaise, et nous rendons compte de différents aspects pragmatiques de l'emploi du $\mathrm{T}$ en contexte. Dans la deuxième, nous passons de la prise de conscience de la valeur pragmatique du $\mathrm{T}$ français à l'intériorisation des ES induits par son emploi. Nous partons ici du principe du bilinguisme subordonné où l'acquisition de la langue étrangère se fait par le biais de la langue maternelle, tout en admettant qu'à un moment de notre vie il est impossible d'apprendre la première indépendamment de la seconde. Nous admettons aussi que la sensibilité d'une langue est aussi importante que sa compréhension. Or, tant qu'on «sent» parfaitement dans sa langue maternelle, même si on ne «comprend» pas tout, dans la langue étrangère bien qu'on «comprenne» beaucoup, on a du mal à «sentir». Pour remédier donc à l'absence de sensibilité à la langue étrangère, de l'obstacle que dans l'apprentissage de cette langue peut poser la sensibilité insistante de la langue maternelle nous en ferons un avantage et nous nous servirons de cette sensibilité déjà existante pour accéder à celle qui nous est étrange(ère). Nous éliminons donc l'influence de la langue maternelle tout en s'en servant, et pour faire sentir les ES et les effets pragmatiques d'emploi de certains $\mathrm{T}$ français (pour le verbe $-l$, en contexte $-k$ ) nous proposerons des expériences linguistiques réalisées en polonais qui produiront des ES proches ou analogues aux ES des temps français. L'objectif de l'enseignant sera donc d'éveiller les dits ES en polonais, d'en faire une description introspective, de les attribuer aux temps français correspondants et de les fixer ${ }^{4}$.

1) Enoncé/Pl $\{\mathrm{Fff}\} \stackrel{\text { éveilleret decrive }}{\longrightarrow} \mathrm{ES} ; 2)$ Enoncé/ $\operatorname{Fr}\left\{\operatorname{Tfr}(l)_{2}^{k}\right\} \stackrel{\text { astribuer et fixer }}{\longleftarrow}$ ES

\subsection{PROSZE WYBACZYĆ SPOŹNIENIE, ALE «MAM ZAPOMNIANY ZEGAREK»}

C'est le premier exercice recommandé. J'annonce cette phrase ${ }^{5}$ en entrant en classe et je regarde l'heure à mon poignet. Après quoi je demande aux étudiants de décrire les ES qu'elle induit et d'évaluer son appropriation. Leur réaction est évidente: „pourquoi il dit qu'il «a sa montre oubliée», alors que je vois qu'il l'a sur

\footnotetext{
${ }^{4}$ Cette technique a été conçue à l'origine pour enseigner les ES des articles (Florczak, 2002), mais ces principes peuvent être appliqués à l'enseignement des ES de certains temps.

${ }^{5}$ Cela peut être d'autres phrases, p.ex: Proszę wybaczyć nieobecność na poprzednich zajęciach, ale mam zlamanq noge / Excusez-moi de ne pas avoir assuré le cour précédent, mais je me suis ( $j^{\prime}$ 'ain) cassé la jambe (tout en n'ayant aucune fracture), qui produiront, suivant leur contexte, des ES analogues à la phrase ci-dessus.
} 
lui". Mon message est donc mal réussi, car il n'est pas adéquat à la situation de communication, et on posera que l'ES qu'il induit correspond à l'ES induit par l'emploi du PC $(\Sigma \alpha)$. Nous remarquerons également qu'en gardant cette construction fort bizarre (car «non-polonaise») l'auditeur sait qu'il aurait fallu dire: «miatem zapomniany zegarek», ce qui correspond à son tour à l'ES induit par l'emploi du $\mathrm{PQP}(\Sigma \beta)$. Nous obtenons donc les équivalences suivantes:

Excusez-moi d'être en retard...

mais j'ai oublié ma montre $\rightarrow \sum \alpha \leftarrow \ldots$ ale «mam zapomniany» zegarek

mais j'avais oublié ma montre $\rightarrow \Sigma \beta \leftarrow \ldots$ ale «miatem zapomniany» zegarek

Pour rendre compte des différences entre les $\Sigma \alpha$ et $\Sigma \beta$ des temps PC i PQP, je me sers donc d'une simple technique de traduction littérale ${ }^{6}$ en soulignant la valeur temporelle du verbe auxiliaire (mieć / być) et en «faisant oublier» la valeur aspectuelle du participe passé ${ }^{7}$. D'où:

J'ai («ma $m$ ») vs J'avais («m i a te $m$ ») attrapé (nabyty) le rhume (katar)

Je me suis («jestem») vs Je m'étais («bytem») foulé (ze skręconq) la cheville (kostkq)

Cette expérience permet de sensibiliser à la valeur pragmatique de l'emploi du temps et aux $\Sigma \alpha$ et $\Sigma \beta$ que l'emploi des PC et PQP produit dans l'esprit de l'auditeur. Elle sensibilise au concept de message non réussi et à la différence entre les concepts de correction de la langue et de message approprié ou efficace.

\subsection{INFÉRENCES CONTEXTUELLES}

L'emploi d'un T en contexte induit des inférences contextuelles qui constituent l'un des composants de l'ES de l'AV analysée. La description de ces inférences, à l'appui des équivalents polonais, pourrait se faire de la manière suivante:

$J$ 'ai attrapé le rhume $\rightarrow$ «mam złapany» | ma voix est encore enrouée $\mid$

J'avais attrapé le rhume $\rightarrow$ "mialem ztapany" | ma voix n'est plus enrouée $\mid$

Ce que j'ai eu froid hier $\rightarrow$ «mam miany" | $j$ 'en tremble encore quand $j$ 'y pense

Ce que j'avais froid hier $\rightarrow$ miany» je n'en tremble plus quand j'y pense

${ }^{6}$ La technique de traduction littérale requiert souvent des équivalents polonais insolites que je n'hésiterai pas à employer même aux dépens de l'infraction de loin abusive aux règles de la langue dont je suis l'usager natif; et ce recours à la langue maternelle peut être critiqué, mais il a ses avantages, car il permet à l'usager de la langue polonaise de s'approcher de la structure conceptuelle de la langue française au moyen dont il dispose. On soulignera pourtant que même si les formes polonaises ci-dessus évoquent les ES p roch es des ES des T français, elles ne leur sont pas synonymes, ne serait-ce que par le fait qu'elles ne sont pas conventionnalisées. Si donc J'ai oublié ma montre induit l'ES décrit à l'aide de «Mam zapomniany zegarek», le premier sera pris pour normal (ن) en français, tandis que le polonais considérera le second comme lui étant absolument étrange(r) (ब) (cf. Turewicz, 1998: 75).

${ }^{7}$ Car tant que mieć et być ont une valeur aspectuelle neutre, les participes passés polonais cidessus sont de fait aspectualisés au perfectif. 
La sélection de telles inférences peut se faire à l'aide des formules suivantes:

de la phrase p je (ne) conclue (pas) que q et/ou il (ne) résulte (pas) que q; et:

en constatant p, on (ne) sait (pas) que/si q et/ou on (ne) suppose (pas) que $q$

qui, formulées telles quelles, peuvent servir aux apprenants polonais à tester les natifs de langue française afin de vérifier les inférences établies. Par exemple:

Elle était belle; $\odot$ on sait qu'elle l'est toujours; $\otimes$ on suppose qu'elle ne l'est plus

Elle avait été belle; $\odot$ on sait qu'elle ne l'est plus; $\odot$ on suppose qu'elle l'est toujours

L'analyse des inférences contextuelles du T français avec son $\mathrm{ES}$ «polonais» semble donc aider à mieux comprendre la valeur pragmatique de l'AV française. Nous soulignons cependant que la description des inférences ci-dessus est très simplifiée et qu'une systématique univoque des inférences purement temporelles n'est pas possible. Ces inférences ne dépendent pas en fait que du temps employé, mais de tout leur interprétant largement compris, soit du verbe même et de son contexte d'emploi ${ }^{8}$. Mais la classification de ces inférences n'est pas notre objectif. En fait, plutôt que de présenter une analyse approfondie du temps français, il s'agit ici de proposer une technique ou plus modestement une astuce didactique qui permettra de faire sentir la particularité de l'AV française aux apprenants polonais dont la langue ne dispose pas de telles formes temporelles. Leur conscience permet de restituer la «normalité» aux emplois «non-étranges» et qui pouvaient être perçus comme tels par la suite de l'assimilation de la temporalité polonaise à la temporalité française, p.ex.:

Q Demain ( $\leftrightarrow$ 'jutro') je suis $(\Sigma \alpha \rightarrow \ll j e s t e m »)$ déjà parti

(-) J'ai ( $\left(\alpha \rightarrow \ll m a m_{*}\right)$ perdu la montre en or depuis longtemps ( $\leftrightarrow$ 'od dawna')

et de sensibiliser aux emplois éventuellement mal appropriés ou inefficaces dans les phrases tout à fait correctes. Nous l'illustrerons à l'aide de l'exemple suivant:

Excuse-moi ma tante, mais

je suis pari vs j'étais parti

où le premier implique: «jestem wyjechany", ce que n'implique pas le second, et leurs emplois entraînent des conséquences différentes. On ne dira pas en fait Je suis parti dans la situation où «nie jestem wyjechany», et l'emploi de je suis parti sera considéré comme acceptable, lorsque, p.ex., en habitant Lódź je téléphone de Poznań pour justifier mon absence à la fête chez ma tante. En revanche, en

${ }^{8}$ P.ex.: je t'ai toujours dit $x \rightarrow$ je n'ai pas changé d'opinion, mais le même PC en cas: je t'ai aimée $\supset$ je ne t'aime plus, $j$ 'ai été malade $\rightarrow$ je ne le suis plus, autre exemple: j'ai voulu $\rightarrow$ je veux toujours, mais dans certaines situations, p.ex. en entrant dans le secrétariat du directeur: Bonjour, je voulais parler à $\mathrm{Mr}$ le directeur ou en m'adressant à la vendeuse du magasin: Je voulais voir ça, «je voulais» n'infère pas du tout Je ne veux plus. (cf. aussi Langacker, 1991: 142, pour connaître et apprendre). 
justifiant cette absence après mon retour à Łódź, on considérera comme mal approprié de dire je suis parti, car je ne peux pas dire à ma tante «jestem wyjechany», et pour justifier mon absence personnellement il faudra que je lui dise «byłem wyjechany» $\leftarrow$ j'étais parti.

La lecture des ES induits par les inférences ci-dessus exige une précision très importante. Il existe en fait des situations où l'on peut employer un même $\mathrm{T}$ pour décrire les événements déroulés sur différents points de l'axe temporel, lorsque leur contexte détermine de manière suffisamment claire que leur emploi ne mène à aucun doute:

Ce matin j'ai oublié de prendre ma montre et j'ai prolongé mon cour de linguistique, mais maintenant (= à Sh de l'après-midi) je l'ai déjà (prise) et je vous lâcherai à l'heure

Hier je suis venu en retard à la fac parce que ma voiture est tombée en panne, mais là $(=$ aujourd'hui) je l'ai déjà réparée et je...

D'où cette remarque importante pour comprendre la nature de l'AV française. Dans un contexte particulier, parler de l'avenir ou du futur n'exclut pas la possibilité d'employer un temps dont l'ES est décrit à l'aide de mam ou jestem. Jestem et mam lexicalisent la forme non-marquée du $\mathrm{T}$ présent par opposition aux formes marquées des $T$ non-présents et, conformément au principe d'économie linguistique ou à la loi du moindre effort, leur emploi sera préféré à l'emploi des formes marquées. Ceci semble remettre en question (d'autres diront: contredire) la validité de notre technique, car le je suis parti de tout à l'heure pourra être dit même en présence de ma tante, sans qu'elle soit étonnée de me voir auprès d'elle. En admettant donc que notre technique exige d'autres spécifications, cela ne déprécie aucunement ses principes généraux, car mam et jestem ne doivent pas forcément signifier jestem et mam ici et maintenant, et les ES «français» qu'ils «réveillent» permettent de mieux sentir la différence entre l'optique de «maintenant» (mam) et d'«alors» (cf. plus haut: action dans et en dehors de son déroulement) qui est aussi caractéristique à la temporalité française:

Cet été j'ai ("mam») été très heureux, j'ai («mam») beaucoup voyagé, j'ai («mam») rencontré ("spot/y/kanych») des gens, j'ai ("mam») fait ("spędzo/a/ny czas na») la fête...

Cet été j'étais («") très heureux, je voyageais ("\#) beaucoup, je rencontrais (《») des gens, je faisais («») la fête...

\subsection{EXERCICES CONTEXTUELS}

Deux types d'exercices sont à réaliser en vue de fixer les ES ainsi découverts. Dans le premier, il s'agit de sensibiliser les apprenants polonais aux ES français envisageables au niveau implicite ou pouvant être attribués aux AV décrites dans un texte rédigé en polonais; l'idéal étant de percevoir ce texte par le biais de la sensibilité temporelle française. Ainsi, on pourrait imaginer que celui qui veut dire 
Nie mogtem z wami zagrać bo skręcilem sobie kostkę a une cheville foulée, ce qui l'obligerait à le dire comme suit: Je n'ai pas pu venir jouer avec vous parce que je me suis foulé la cheville. Le choix de ce T n'est pas contraignant en termes de contraintes grammaticales, et l'objectif de l'exercice n'est pas de «mettre le T correct», mais de justifier la validité de son emploi par son interprétant largement compris, dont: situation de communication, inférences contextuelles envisageables et locuteur.

\subsection{TESTS DE COMMUTATION}

Une fois les ES décortiqués, les textes polonais traduits et les ES lexicalisés par les temps correspondants, on passe à l'exercice suivant qui consiste à substituer au $T$ choisi un autre temps et à en décrire les conséquences. Dans cet exercice on fait donc justifier le choix d'un $\mathrm{T}$ en même temps que le non choix d'un autre.

A partir de là, quelques possibilités sont envisageables. L'ES induit par le T employé reste en contradiction avec le contexte ou la situation de communication, et le choix d'un $\mathrm{T}$ étant exclu, le choix de l'autre est nécessaire. Dans ce cas, l'emploi du T est validé en termes de non-acceptabilité, et l'évaluation de son emploi se fait en termes d'opposition entre emploi possible et nécessaire, soit: $\diamond$ vs $\square$. P.ex.:

Je lui ai rendu le livre que je lui $\square$ avais («i)emprunté

Cet été je $\diamond$ voyageais (j'ai voyagé) beaucoup. je rencontrais (j'ai rencontré) des gens

Il se peut aussi que l'emploi de différents temps soit acceptable. Dans ce cas, le choix du $T$ est à la fois libre, parce que seul le locuteur le détermine, et contraignant, dans la mesure où il l'engage à assumer la responsabilité de l'ES qu'il induit. La validation de son emploi se fait ici en termes de n on-effic a c ité, et les apprenants sont invités à expliquer les ES et les inférences contextuelles du T employé, ainsi que des $\mathrm{T}$ dont les ES et inférences contextuelles envisageables les ont empêchés de le faire. Vu que le choix du T ne résulte ici que de l'ES visé par le locuteur, l'évaluation de son emploi se fait en termes d'emplois «réussis vs mal réussis» (:) vs $(2)$, en vérifiant si l'ES induit correspond à l'intention de son émetteur $(v=\varpi$ ou $v \neq \varpi)$. Pour illustrer ces propos avec un exercice pratique, prenons la phrase polonaise de tout à l'heure qui sans un contexte plus précis ne permet pas de faire un choix univoque entre le $\Sigma \alpha$ du PC et le $\Sigma \beta$ du PQP et peut être traduite de deux manières, les deux étant correctes:

Nie mogtem z wami zagrać bo skr ęcile m sobie $(\rightarrow \Sigma \alpha$ ou $\Sigma \beta)$ kostkę

I) Je n'ai pas pu jouer avec vous parce que je me su is ( $\Sigma a \rightarrow$ umam») foulé la cheville

2) Je ne pouvais pas jouer parce que je m'ét a is ( $\Sigma \beta \rightarrow$ "miatem») foulé la cheville?

${ }^{9}$ L'emploi du PC dans je me suis foulé entraîne l'emploi du PC dans je n'ai pas pu, car qui a une cheville foulée n'a pas la capacité de jouer, et qui a cette capacité ne peut pas avoir de cheville foulée. 
Mais chacune des traductions françaises induira une autre inférence et la validation de l'emploi du $T$, i.e. la vérification de la compréhension de son $\mathrm{ES}$ consistera à décrire ces inférences. L'emploi du PC dans 1) peut être interprété comme la justification d'un footballeur dont le pied est encore dans le plâtre, tandis que la phrase 2) peut être interprétée comme un fragment de conversation sur la manière dont l'équipe aurait pu gagner $(\rightarrow \mid n$ 'a pas gagné $\mid)$ un match très important l'année dernière.

En somme, pour le choix du $\mathrm{T}$ et pour expliquer sa validité nous opérons tantôt dans la perspective sémasiologique, pour voir ce que nous pouvons dire lorsque:

Proszę wybaczyć mi to spóźnienie, ale...

a) nie wiedziatem która godzina, bo zapomniatem zegarka $\stackrel{\text { sémas. }}{\longrightarrow}(\Sigma a \leftarrow c f .1)$ je ne savais pas quelle heure il était parce que..

b) musialem się wrócić do domu, bo zapomnialem zegarka $\stackrel{\text { sémas. }}{\longrightarrow}(\Sigma \beta \leftarrow c f .2)$ j'ai du retourner chez moi parce que...

tantôt onomasiologique, pour décider de ce que l'on doit dire quand:

Excusez-moi d'être en retard, mais...

1) () j'ai oublié ma montre $\stackrel{\text { onomas. }}{\longleftarrow}$ je n'ai pas ma montre sur moi, car «mam zapomniany,

(ㄱ'avais oublié ma montre $(\vartheta \neq \varpi)$

2) $\odot$ j'avais oublié ma montre $\longleftarrow$ onomas. nianyw

@ j'ai oublié ma montre $(\nu \neq \varpi)$

Et l'objectif de ces exercices est de faire prendre conscience des ES induits par certains $\mathrm{T}$ en contexte de manière à ce que nos apprenants les emploient conformément à leurs intentions, tout en assumant la responsabilité de leurs emplois réussis ou non.

\section{CONCLUSION}

La nature du système temporel français est évidemment beaucoup plus complexe que les valeurs décrites ci-dessus. Outre l'aspect pragmatique il en existe bien d'autres tout aussi importants, sinon plus, et qui devraient être pris en compte dans une analyse plus complète. D'ailleurs, même notre analyse pragmatique est en elle-même fragmentaire et une description plus rigide devrait rendre compte de tout un ensemble de facteurs que nous n'avons pas analysés, comme, p.ex., la valeur lexicale (ordre du procès ou sens) du verbe employé, la présence de circonstanciels de temps, etc. Nous soulignons donc qu'elle ne peut être en aucun cas considérée comme analyse exhaustive de la valeur pragmatique de l'AV française, et il convient $d$ 'avertir contre une extrapolation quelconque des règles inférencielles proposées à tous les cas d'emploi du temps. 
Mais l'objectif de notre travail a été ailleurs. Il s'agissait en fait de proposer une procédure didactique particulière qui permettrait d'éviter les écueils des méthodes d'enseignement traditionnelles, qui, basées sur différents types d'exercices bomés à des contextes isolés, peuvent fausser les véritables valeurs de l'AV française et les écueils des explications linguistiques dont les théories proliférantes et souvent non homogènes ne font qu'effrayer ceux qui n'y sont pas initiés et semblent servir en fait à expliquer le temps français à ceux qui s'en servent déjà. Tout en adaptant donc les outils du linguiste aux besoins du didacticien du FLE, nous proposons une technique d'enseignement construite spécialement pour les apprenants polonais dont la langue n'exprime pas ces valeurs à la manière française, et notre enjeu est d'éveiller les valeurs qu'ils n'ont pas au moyen de ce dont ils disposent. Notre approche doit être considérée comme une simple astuce créée au service de l'enseignant et de l'apprenant en vue de pourvoir le premier d'une technique de sensibilisation de ses élèves à certaines valeurs de l'AV française et le second d'une base conceptuelle et des outils d'analyse dont il pourra se servir pour mieux connaître la temporalité française dont la compréhension pose encore à tout apprenant polonais de nombreux problèmes.

\section{BIBLIOGRAPHIE}

Ducrot, O. (1972), Dire et ne pas dire. Paris: Hermann.

Florc zak, J. (2002), De l'approche pragmatique de l'article, ou comment la pragmatique peut aider à comprendre et/ou à enseigner le système de l'article français, in: Points communs: linguistique, traductologie, glottodidactique. Lódź, 37-56.

Gross, G. (2002), Recherches théoriques et enseignement des langues, in: Points communs: linguistique, traductologie, glottodidactique, Lódź, 88-101.

Langacker, R. (1991), Noms et verbes, trad. C. Vandeloise, in: Communication 53. Sémantique cognitive. Paris, 103-145.

Śtichaucr, J. (2002), Aspect verbal - une approche contrastive est-elle pertinente, in: Points communs: linguistique, traductologie, glottodidactique. Łódź, 269-274.

Tokar z, M. (1993), Elementy pragmatyki logicznej. Warszawa: PWN.

Turewicz, K. (1998), Profil a kategorie gramatyczne, in: Profilowanie w jezyku iw tekscie. Lublin, 63-77.

Wilczyńska, W. (1989), Apprivoiser les articles et les temps. Poznań: UAM. 